\title{
Electron/phonon coupling in group-IV transition-metal and rare-earth nitrides
}

A B Mei, A Rockett, Lars Hultman, I Petrov and J E Greene

\section{Linköping University Post Print}

\section{Tweet}

N.B.: When citing this work, cite the original article.

Original Publication:

A B Mei, A Rockett, Lars Hultman, I Petrov and J E Greene, Electron/phonon coupling in group-IV transition-metal and rare-earth nitrides, 2013, Journal of Applied Physics, (114), 19, 193708.

http://dx.doi.org/10.1063/1.4832778

Copyright: American Institute of Physics (AIP) http://www.aip.org/

Postprint available at: Linköping University Electronic Press

http://urn.kb.se/resolve?urn=urn:nbn:se:liu:diva-102778 


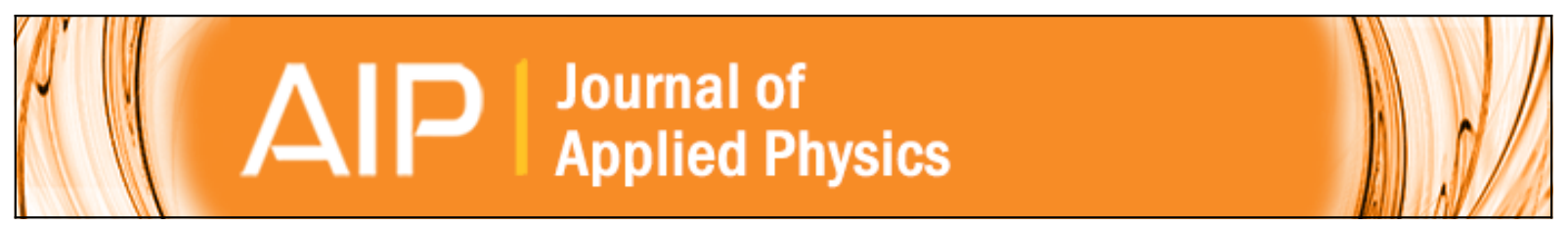

Electron/phonon coupling in group-IV transition-metal and rare-earth nitrides

A. B. Mei, A. Rockett, L. Hultman, I. Petrov, and J. E. Greene

Citation: Journal of Applied Physics 114, 193708 (2013); doi: 10.1063/1.4832778

View online: http://dx.doi.org/10.1063/1.4832778

View Table of Contents: http://scitation.aip.org/content/aip/journal/jap/114/19?ver=pdfcov

Published by the AIP Publishing

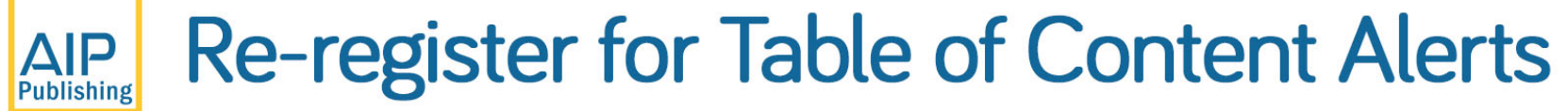

Create a profile.

Sign up today! 


\title{
Electron/phonon coupling in group-IV transition-metal and rare-earth nitrides
}

\author{
A. B. Mei, ${ }^{1}$ A. Rockett, ${ }^{1}$ L. Hultman, ${ }^{2}$ I. Petrov,${ }^{1,2}$ and J. E. Greene ${ }^{1,2}$ \\ ${ }^{1}$ Departments of Materials Science, Physics, and the Materials Research Laboratory, \\ University of Illinois, 104 South Goodwin, Urbana, Illinois 61801, USA \\ ${ }^{2}$ Thin Film Physics Division, Department of Physics (IFM), Linköping University, \\ SE-58183 Linköping, Sweden
}

(Received 5 September 2013; accepted 6 November 2013; published online 20 November 2013)

Transport electron/phonon coupling parameters and Eliashberg spectral functions $\alpha_{\mathrm{tr}}{ }^{2} \mathrm{~F}(\hbar \omega)$ are determined for group-IV transition-metal (TM) nitrides TiN, $\mathrm{ZrN}$, and $\mathrm{HfN}$, and the rare-earth (RE) nitride $\mathrm{CeN}$ using an inversion procedure based upon temperature-dependent $(4<\mathrm{T}<300 \mathrm{~K})$ resistivity measurements of high-crystalline-quality stoichiometric epitaxial films grown on $\mathrm{MgO}(001)$ by magnetically-unbalanced reactive magnetron sputtering. Transport electron/phonon coupling parameters $\lambda_{\mathrm{tr}}$ vary from 1.11 for $\mathrm{ZrN}$ to 0.82 for $\mathrm{HfN}, 0.73$ for TiN, and 0.44 for CeN. The small variation in $\lambda_{\text {tr }}$ among the TM nitrides and the weak coupling in $\mathrm{CeN}$ are consistent with measured superconducting transition temperatures $10.4(\mathrm{ZrN}), 9.18(\mathrm{HfN}), 5.35(\mathrm{TiN})$, and $<4 \mathrm{~K}$ for $\mathrm{CeN}$. The Eliashberg spectral function describes the strength and energy spectrum of electron/phonon coupling in conventional superconductors. Spectral peaks in $\alpha^{2} \mathrm{~F}(\hbar \omega)$, corresponding to regions in energy-space for which electrons couple to acoustic $\hbar \omega_{\text {ac }}$ and optical $\hbar \omega_{\text {op }}$ phonon modes, are centered at $\hbar \omega_{\mathrm{ac}}=33$ and $\hbar \omega_{\mathrm{op}}=57 \mathrm{meV}$ for TiN, 25 and $60 \mathrm{meV}$ for $\mathrm{ZrN}, 18$ and $64 \mathrm{meV}$ for $\mathrm{HfN}$, and 21 and $39 \mathrm{meV}$ for $\mathrm{CeN}$. The acoustic modes soften with increasing cation mass; optical mode energies remain approximately constant for the TM nitrides, but are significantly lower for the RE nitride due to a lower interatomic force constant. Optical/acoustic peak-intensity ratios are $1.15 \pm 0.1$ for all four nitrides, indicating similar electron/phonon coupling strengths $\alpha_{\mathrm{tr}}(\hbar \omega)$ for both modes. (C) 2013 AIP Publishing LLC. [http://dx.doi.org/10.1063/1.4832778]

\section{INTRODUCTION}

Superconductivity, the ability to conduct electrons without resistance, occurs in a variety of materials. One of the earliest conventional superconductors discovered to have a relatively high transition temperature $\left(\mathrm{T}_{\mathrm{c}}\right)$ is the group $-\mathrm{V}$ $\mathrm{NaCl}$-structure transition-metal (TM) nitride $\mathrm{NbN}$ with $\mathrm{T}_{\mathrm{c}}=16 \mathrm{~K} .{ }^{1}$ Since then, conventional superconductors have found a broad range of device applications including microresonator detectors, ${ }^{2,3}$ waveguide resonators, ${ }^{4}$ accelerator cavities, ${ }^{5}$ coil magnets, ${ }^{6}$ qubit computing, ${ }^{7}$ Josephson junctions in quantum interference devices, ${ }^{8}$ hot electron bolometers,${ }^{9}$ single-photon detectors, ${ }^{10}$ and THz mixers. ${ }^{11}$

Recently, there has been increased interest in group-IV TM nitrides. ${ }^{12-15}$ Here, we present a systematic investigation of the transport Eliashberg coupling function $\alpha_{\mathrm{tr}}{ }^{2} \mathrm{~F}(\hbar \omega)$, an energy-resolved measure of the electron/phonon coupling strength $\alpha_{\mathrm{tr}}^{2}(\hbar \omega)$ and the phonon density-of-states $\mathrm{F}(\hbar \omega)$, for the epitaxial TM nitrides $\mathrm{TiN}, \mathrm{ZrN}$, and $\mathrm{HfN}$, as well as the group-IV rare-earth (RE) nitride CeN. Apart from a geometric factor which favors backscattered electrons ${ }^{15}$ the transport Eliashberg function contains, together with the Coulomb pseudopotential $\mu^{*},{ }^{16}$ all information pertaining to phonon-mediated superconductivity. As a result, properties such as temperature- and frequency-dependent energy gaps are readily acquired from $\alpha_{\mathrm{tr}}{ }^{2} \mathrm{~F}(\hbar \omega)$ through the Eliashberg gap equations. ${ }^{17,18}$

We obtain $\alpha_{\mathrm{tr}}{ }^{2} \mathrm{~F}(\hbar \omega)$ from an inversion procedure based on temperature-dependent resistivity measurements, between
4 and $300 \mathrm{~K}$, of high-crystalline-quality stoichiometric epitaxial $\mathrm{TiN}, \mathrm{ZrN}$, $\mathrm{HfN}$, and $\mathrm{CeN}$ layers grown on $\mathrm{MgO}(001)$. For all compounds, the coupling strength $\alpha_{\mathrm{tr}}{ }^{2} \mathrm{~F}(\hbar \omega)$ is determined to be approximately uniform over energy $\hbar \omega$, indicating that $\alpha_{\mathrm{tr}}{ }^{2} \mathrm{~F}(\hbar \omega)$ effectively probes the phonon density-of-states. This is, to our knowledge, the first experimental determination of $\alpha^{2} \mathrm{~F}(\hbar \omega)$ for TiN, HfN, and CeN.

\section{EXPERIMENTAL PROCEDURE}

Single-crystal stoichiometric $\mathrm{TiN}, \mathrm{ZrN}, \mathrm{HfN}$, and $\mathrm{CeN}$ layers, with metal/nitrogen ratios $\mathrm{Me} / \mathrm{N}=1.00 \pm 0.03$ and thicknesses $\mathrm{t} \geq 70 \mathrm{~nm}$, are grown on $\mathrm{MgO}(001)$ in a loadlocked ultra-high-vacuum (UHV) magnetically-unbalanced stainless-steel dc magnetron sputter deposition system described in detail by Petrov et al. ${ }^{19}$ The composition and structure of as-deposited samples are determined using a combination of Rutherford backscattering spectrometry (RBS), high-resolution x-ray diffraction (HR-XRD), and cross-sectional transmission electron microscopy (XTEM). Details regarding film growth and characterization are described in Ref. 19 for TiN, Ref. 20 for ZrN, Ref. 21 for $\mathrm{HfN}$, and Ref. 22 for CeN. All films have the $\mathrm{NaCl}$ structure and grow with a cube-on-cube epitaxial relationship to the substrate, $(001)_{\text {nitride }} \|(001)_{\mathrm{MgO}}$ and $[100]_{\text {nitride }} \|[100]_{\mathrm{MgO}}$. Relaxed lattice constants are 0.4240 (TiN), $0.4574(\mathrm{ZrN})$, $0.4524(\mathrm{HfN})$, and $0.5021 \mathrm{~nm}(\mathrm{CeN})$. In-plane $\xi_{\|}$and out-ofplane $\xi_{\perp}$ X-ray coherence lengths are 86 and $142 \mathrm{~nm}$ for TiN, 18 and $161 \mathrm{~nm}$ for $\mathrm{ZrN}, 22$ and 185 for $\mathrm{HfN}$, and 7 and 
$26 \mathrm{~nm}$ for CeN. The results indicate that the films are of high crystalline quality with low mosaicity. ${ }^{23}$

Temperature-dependent resistivities between 4 and $300 \mathrm{~K}$ are obtained using a Quantum Design physical property measurement system. Electrical contacts are fabricated in an FEI Strata DB-235 focused ion-beam system by $\mathrm{Ga}^{+}$ ion-etching four 2- $\mu$ m-diameter holes, in the van der Pauw geometry, ${ }^{24}$ and then filling the holes with Pt without breaking vacuum. All contacts are tested for ohmic behavior. Current and voltage measurements are iterated through different contact pairs to account for geometric effects.

\section{RESULTS AND DISCUSSION}

Temperature-dependent resistivities $\rho(\mathrm{T})$ between 4 and $300 \mathrm{~K}$ are plotted in Figure 1. Room-temperature resistivity $\rho_{300 \mathrm{~K}}$ values are $12.9(\mathrm{TiN}), 12.0(\mathrm{ZrN}), 14.2(\mathrm{HfN})$, and $69 \mu \Omega$-cm $(\mathrm{CeN})$; all essentially equal to, or better, than corresponding values for bulk crystals: 11.07 (TiN), 11.52 $(\mathrm{ZrN})$, and $33 \mu \Omega-\mathrm{cm}(\mathrm{HfN}){ }^{25,26} \mathrm{We}$ are unaware of published results for bulk CeN. $\rho_{300 \mathrm{~K}}$ values for polycrystalline films are typically $\sim 2$ to $10^{6}$ times higher; $\rho_{300 \mathrm{~K}}$ for polycrystalline TiN ranges from 20 to $200 \mu \Omega-\mathrm{cm},{ }^{27,28}$ polycrystalline $\mathrm{ZrN}$ from 23 to $2000 \mu \Omega-\mathrm{cm},{ }^{29-31}$ polycrystalline HfN from 225 to $800 \mu \Omega-\mathrm{cm},{ }^{32}$ and polycrystalline CeN from 460 to $>2 \times 10^{8} \mu \Omega-\mathrm{cm} .^{33-35}$ The low resistivity values of our epitaxial films are an additional indicator of high structural quality.

The resistivity of all films increases approximately linearly with temperature between 100 and $300 \mathrm{~K}$, indicating metallic conduction with a carrier mobility that is controlled by phonon scattering. Over this range, the temperature coefficients of resistivity, defined as TCR $=\left(\rho_{300 \mathrm{~K}-} \rho_{100 \mathrm{~K}}\right) / \Delta \mathrm{T}$, are $5.0 \times 10^{-8}$ for $\mathrm{TiN}, 5.6 \times 10^{-8}$ for $\mathrm{ZrN}, 4.0 \times 10^{-8}$ for $\mathrm{HfN}$, and $16.0 \times 10^{-8} \Omega-\mathrm{cm} \mathrm{K}^{-1}$ for $\mathrm{CeN}$.

At temperatures $\mathrm{T}$ below $30 \mathrm{~K}$, phonon scattering is negligible and the resistivity is primarily determined by defect and impurity scattering. Figure 1 shows that $\rho(\mathrm{T})$ for the four nitrides saturates at residual resistivities $\rho_{\mathrm{o}}=2.08(\mathrm{TiN}), 0.78$ $(\mathrm{ZrN}), 3.5(\mathrm{HfN})$, and $29 \mu \Omega-\mathrm{cm}(\mathrm{CeN})$. In metallic conductors, the residual resistivity ratio $\mathrm{RRR}=\rho_{300 \mathrm{~K}} / \rho_{\mathrm{o}}$ is used as a metric of crystalline quality. For the group-IV nitrides under

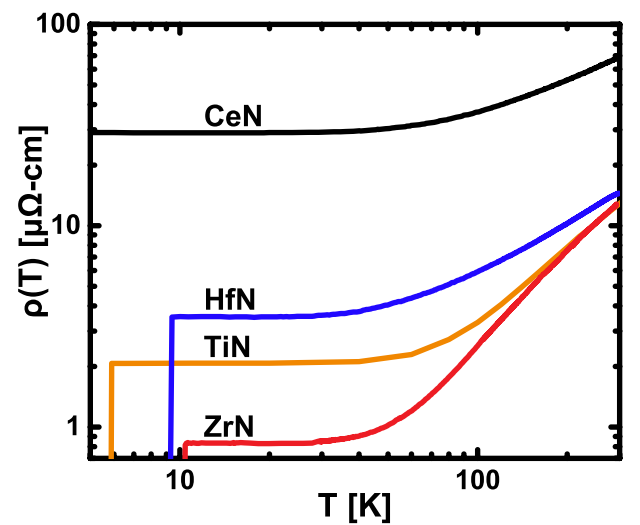

FIG. 1. Temperature-dependent resistivity $\rho(\mathrm{T})$ of single-crystal $\mathrm{TiN}, \mathrm{ZrN}$, $\mathrm{HfN}$, and $\mathrm{CeN}$ layers grown on $\mathrm{MgO}(001)$ by magnetically-unbalanced reactive magnetron sputtering. TiN data from Ref. 19, $\mathrm{ZrN}$ from Ref. 20, HfN from Ref. 21, and CeN from Ref. 22. investigation, RRR values are relatively high: 6.2 (TiN), 15 $(\mathrm{ZrN}), 4(\mathrm{HfN})$, and $2.4(\mathrm{CeN})$. As a result, scattering events are independent and the resistivity over the entire measured temperature range is described by Matthiessen's rule $\rho(\mathrm{T})=\rho_{\mathrm{o}}+\rho_{\mathrm{ph}}(\mathrm{T})$, in which $\rho_{\mathrm{ph}}(\mathrm{T})$ is the phonon-scattering contribution to resistivity.

Within the relaxation time approximation, $\rho_{\mathrm{ph}}(\mathrm{T})$ can be expressed as ${ }^{36}$

$$
\rho_{\mathrm{ph}}(\mathrm{T})=\frac{4 \pi}{\omega_{\mathrm{p}}^{2}\langle\tau\rangle}=\frac{\mathrm{m}^{*}}{\mathrm{ne}^{2}\langle\tau\rangle}
$$

$\omega_{\mathrm{p}}$ is the unscreened plasma frequency and $\mathrm{n}, \mathrm{e}$, and $\mathrm{m}^{*}$ are the electron concentration, charge, and effective mass, respectively. The rate $\langle\tau\rangle^{-1}$ at which electrons scatter from phonons is given by: ${ }^{37}$

$$
\langle\tau\rangle^{-1}=\pi \int_{0}^{\hbar \omega_{\max }} \frac{\hbar \omega}{\mathrm{k}_{\mathrm{B}} \mathrm{T}} \operatorname{csch}\left(\frac{\hbar \omega}{2 \mathrm{k}_{\mathrm{B}} \mathrm{T}}\right) \alpha_{\mathrm{tr}}^{2}(\hbar \omega) \mathrm{F}(\hbar \omega) \mathrm{d} \hbar \omega,
$$

in which $\mathrm{k}_{\mathrm{B}}$ is the Boltzmann constant, $\alpha_{\mathrm{tr}}{ }^{2}(\hbar \omega)$ is the square of the transport electron/phonon coupling function, $\mathrm{F}(\hbar \omega)$ is the phonon density-of-states, and $\hbar \omega_{\max }$ is the energy of the highest phonon mode. The product $\alpha_{\mathrm{tr}}^{2}(\hbar \omega) \mathrm{F}(\hbar \omega)$ is the transport Eliashberg spectral function. ${ }^{38}$

In the high-temperature limit $\left(\theta_{\mathrm{D}} / \mathrm{T} \ll 10\right.$; the Debye temperature $\theta_{\mathrm{D}}$ for metal nitrides ranges from 400 to $600 \mathrm{~K}$ (Ref. 26)), scattering is elastic ${ }^{39}$ and the scattering rate reduces to ${ }^{16}$

$$
\langle\tau\rangle^{-1}=\frac{2 \pi \mathrm{k}_{\mathrm{B}} \mathrm{T}}{\hbar} \lambda_{\mathrm{tr}}
$$

Thus, $\rho$ increases linearly with temperature $\mathrm{T}$ at a rate proportional to the transport electron/phonon coupling parameter $\lambda_{\text {tr }}$ (Ref. 51) and inversely proportional to the square of the unscreened plasma frequency $\omega_{\mathrm{p}}$ (see Eq. (1)). In order to determine $\lambda_{\text {tr }}$, the plasma frequency $\omega_{\mathrm{p}}$ is obtained from first principles density functional theory (DFT) band-structure calculations ${ }^{40}$ by integrating $\mathrm{v}(\mathrm{k})$, the group velocity of electrons at state $\mathrm{k}$, over the Brillouin zone using the relationship ${ }^{41}$

$$
\omega_{\mathrm{p}}^{2}=\frac{\mathrm{e}^{2}}{\pi^{2} \hbar} \int \mathrm{dk} \nabla_{\mathrm{k}} \mathrm{v}(\mathrm{k}) \mathrm{f}(\mathrm{k})
$$

in which $f(k)$ is the Fermi-Dirac occupation probability function.

For the group-IV TM nitrides, we obtain $\omega_{\mathrm{p}}$ values which increase with the cation atomic number $\mathrm{Z}$ from 7.7 for TiN to 8.9 for $\mathrm{ZrN}$ and $9.1 \mathrm{eV}$ for $\mathrm{HfN}$; for the rare-earth (RE) nitride $\mathrm{CeN}, \omega_{\mathrm{p}}=3.3 \mathrm{eV}$, less than half the average $\omega_{\mathrm{p}}$ value of the nitrides. The transport electron/phonon coupling parameters $\lambda_{\text {tr }}$ are obtained from Eqs. (1) and (3), combined with the measured TCR values, as $0.73(\mathrm{TiN}), 1.11(\mathrm{ZrN})$, $0.82(\mathrm{HfN})$, and $0.44(\mathrm{CeN})$. The small variation in $\lambda_{\text {tr }}$ among the TM nitrides and the weak coupling in $\mathrm{CeN}$ are consistent with superconducting transition temperatures which range from $10.4 \mathrm{~K}$ for $\mathrm{ZrN}$ to $9.18 \mathrm{~K}$ for $\mathrm{HfN}$ and $5.35 \mathrm{~K}$ for TiN; superconductivity is not observed above $4 \mathrm{~K}$ for $\mathrm{CeN}$. 
The transport Eliashberg spectral functions for TiN, $\mathrm{ZrN}, \mathrm{HfN}$, and $\mathrm{CeN}$ are developed by first discretizing the integral in Eq. (2) into a geometric series of superimposed Einstein modes ${ }^{42}$

$$
\rho_{\mathrm{ph}}(\mathrm{T})=\frac{4 \pi \mathrm{m}^{*}}{\mathrm{ne}^{2}} \sum_{\mathrm{k}} \alpha^{2} \mathrm{~F}\left(\hbar \omega_{\mathrm{k}}\right) \frac{\mathrm{x}_{\mathrm{k}} \mathrm{e}^{\mathrm{x}_{\mathrm{k}}}}{\left(\mathrm{e}^{\mathrm{x}_{\mathrm{k}}}-1\right)^{2}},
$$

in which $\mathrm{x}_{\mathrm{k}}=\hbar \omega_{\mathrm{k}} / \mathrm{k}_{\mathrm{B}} \mathrm{T}$, and fitting $\rho(\mathrm{T})$ to Matthiessen's rule using the largest number of Einstein components at spacings $\omega_{\mathrm{k}-1} / \omega_{\mathrm{k}}$ which preserve convergence stability, $\omega_{\mathrm{k}-1} / \omega_{\mathrm{k}}$ $\geq 1.57$. The procedure is illustrated graphically in Figure 2 , which shows the $\mathrm{ZrN}$ temperature-dependent resistivity $\rho(\mathrm{T})$ between 10 and $300 \mathrm{~K}$ multiplied by a factor of $1 / \mathrm{T}$ (dotted red curve) in order to highlight resistivity contributions due to defect and phonon scattering. The normalized residual-resistivity $\rho_{\mathrm{o}} / \mathrm{T}$ (dashed black line) simply decays as $1 / \mathrm{T}$, while normalized Einstein components, corresponding to electron/phonon scattering, increase with temperature (black solid lines). Multiple Einstein modes are necessary to reproduce the experimental $\rho(\mathrm{T})$ curve. The calculated normalized temperature-dependent resistivity, which includes the normalized residual resistivity $\rho_{\mathrm{o}} / \mathrm{T}$ and normalized phonon contributions from each of the Einstein modes, is plotted in Figure 2(a) as the orange solid line. Residuals R from a typical $\rho(\mathrm{T})$ fit for $\mathrm{ZrN}$ are shown in Figure 2(b); the agreement is excellent to within experimental uncertainty.

Transport Eliashberg spectral functions $\alpha_{\mathrm{tr}}^{2} \mathrm{~F}(\hbar \omega)$ are generated from 200 fits, including the orange $\rho(\mathrm{T}) / \mathrm{T}$ curve shown in Figure 2, which consist of approximately ten Einstein modes each using different starting values for $\hbar \omega_{1}$, the energy of the first Einstein mode. The energies of the remaining modes, for a particular fit, are defined by $\hbar \omega_{1}$ following the

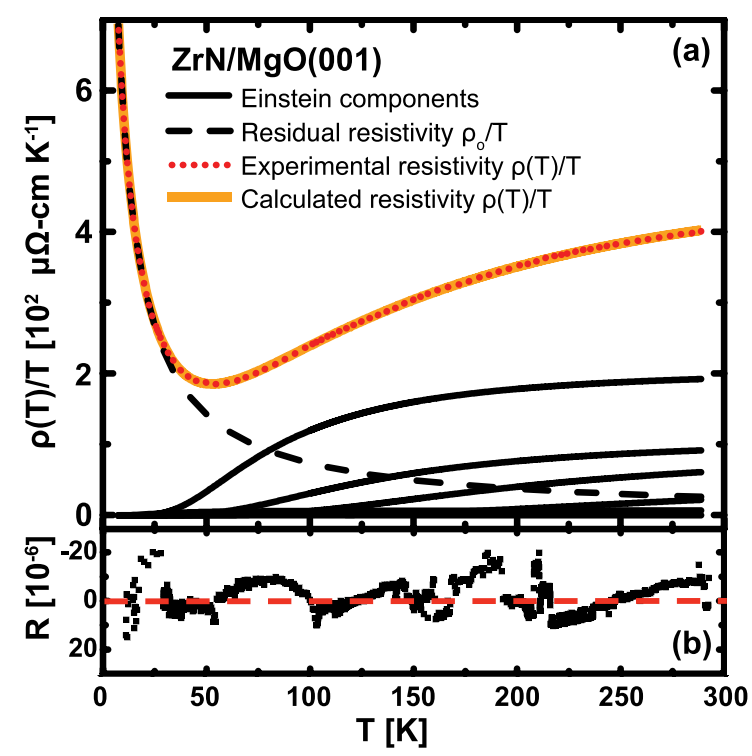

FIG. 2. (a) Measured temperature-dependent resistivity $\rho(\mathrm{T})$ of epitaxial $\mathrm{ZrN} / \mathrm{MgO}(001)$ multiplied by a factor of $1 / \mathrm{T}$ (red dotted line) to highlight contributions due to defect and phonon scattering. The black dashed line is the normalized residual resistivity, $\rho_{\mathrm{o}} / \mathrm{T}$. Each solid black line corresponds to a normalized Einstein mode of known amplitude and energy. The solid orange curve is the calculated total normalized $\mathrm{ZrN}$ resistivity $\rho(\mathrm{T}) / \mathrm{T}$, for which $\rho(\mathrm{T})=\rho_{\mathrm{o}}+\rho_{\mathrm{ph}}(\mathrm{T})$. (b) Residuals R, the difference between measured and calculated resistivities, as a function of temperature $\mathrm{T}$. stability criteria $\omega_{\mathrm{k}-1} / \omega_{\mathrm{k}}=1.57$. Each Einstein mode contributes a discrete point, with coordinates specified by the mode's amplitude and energy, to the spectral function. As a result, 200 fits, with ten Einstein modes each, yield 2000 data points, thus resulting in a transport spectral function curve which is quasi-continuous. $\alpha_{\mathrm{tr}}{ }^{2} \mathrm{~F}(\hbar \omega)$ for $\mathrm{TiN}, \mathrm{ZrN}, \mathrm{HfN}$, and $\mathrm{CeN}$, obtained as described above, are plotted in Figure 3 as a function of vibrational energy $\hbar \omega$. The intensity of $\alpha_{\mathrm{tr}}{ }^{2} \mathrm{~F}(\hbar \omega)$ at a given energy $\hbar \omega$ corresponds to the population of phonon modes at that energy weighted by $\alpha_{\mathrm{tr}}^{2}(\hbar \omega)$, the square of their interaction strengths with electrons.

The CeN transport Eliashberg spectral function $\alpha_{\mathrm{tr}}{ }^{2} \mathrm{~F}(\hbar \omega)$ exhibits features centered at $21 \pm 2$ and $40 \pm 3 \mathrm{meV}$, indicating that electrons in $\mathrm{CeN}$ scatter primarily from two groups of phonons. The lower-energy peak stems from electron/acoustic-phonon scattering and the higher-energy peak from electron/optical-phonon scattering. While this is the first experimental report of phonon behavior in CeN, DFT calculations ${ }^{43}$ indicate that peaks in $\mathrm{F}(\hbar \omega)$, corresponding to acoustic and optical phonon modes, occur at 21 and $39 \mathrm{meV}$, in excellent agreement with the two features observed here.

The $\mathrm{ZrN}$ and HfN transport Eliashberg spectral functions also exhibit two features each, corresponding to acoustic and optical phonon scattering. For $\mathrm{ZrN}$, the peaks are centered at energies $\hbar \omega_{\mathrm{ac}}=25 \pm 2$ and $\hbar \omega_{\mathrm{op}}=60 \pm 6 \mathrm{meV}$; for $\mathrm{HfN}$, at $\hbar \omega_{\mathrm{ac}}=18 \pm 2$ and $\hbar \omega_{\mathrm{op}}=64 \pm 6 \mathrm{meV}$. These results are consistent with vibrational energies obtained from $\mathrm{ZrN}$ and HfN Raman spectroscopy $\left(\hbar \omega_{\mathrm{ac}}=21\right.$ and $\hbar \omega_{\mathrm{op}}=62 \mathrm{meV}$ for $\mathrm{ZrN}^{43}$ and $\hbar \omega_{\mathrm{ac}}=17$ and $\hbar \omega_{\mathrm{op}}=65 \mathrm{meV}$ for $\mathrm{HfN}^{44}$ ) as well as from neutron scattering measurements $\left(\hbar \omega_{\mathrm{ac}}=25\right.$ and $\hbar \omega_{\mathrm{op}}=65 \mathrm{meV}$ for $\mathrm{ZrN}$ (Ref. 45) and $\hbar \omega_{\mathrm{ac}}=18$ and $\hbar \omega_{\mathrm{op}}=62 \mathrm{meV}$ for HfN (Ref. 46)).

For TiN, we obtain a single broad feature centered at $\sim 42 \mathrm{meV}$, which is well fit with two Gaussian functions yielding a low-energy peak at $\hbar \omega_{\mathrm{ac}}=33 \pm 5 \mathrm{meV}$ and a higher-energy peak at $\hbar \omega_{\mathrm{op}}=57 \pm 13 \mathrm{meV}$. These findings are in reasonable agreement with those from Raman measurements, for which $\hbar \omega_{\mathrm{ac}}=32$ and $\hbar \omega_{\mathrm{op}}=68 \mathrm{meV}^{48}$

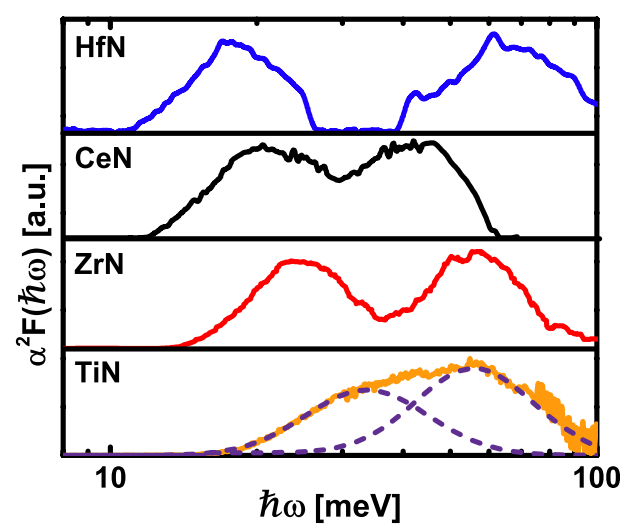

FIG. 3. Transport Eliashberg spectral functions $\alpha_{\mathrm{tr}}{ }^{2} \mathrm{~F}(\hbar \omega)$, obtained from temperature-dependent $(4 \leq \mathrm{T} \leq 300 \mathrm{~K})$ resistivity measurements using an inversion procedure, for single-crystal $\mathrm{TiN}, \mathrm{ZrN}, \mathrm{CeN}$, and $\mathrm{HfN}$ layers grown on $\mathrm{MgO}(001)$ substrates. The TiN spectrum is fit with two Gaussian functions (dashed lines). The lower-energy peak in each spectrum arises from electron/acoustic-phonon coupling while the higher-energy peak is from electron/optical-phonon coupling. 
and neutron scattering experiments, $\hbar \omega_{\mathrm{ac}}=37$ and $\hbar \omega_{\text {op }}=74 \mathrm{meV}^{49}$

In binary compounds, each atom within the primitive unit cell contributes three phonon modes (one for each degree of freedom) to the phonon density-of-states $\mathrm{F}(\hbar \omega)$ such that the total density of normal modes, for a large number $\mathrm{N}$ of unit cells, is $6 \mathrm{~N}$. Compounds composed of atoms with significant mass differences exhibit two groups of phonons: the lower-energy group corresponds to acoustic phonon modes and the higher-energy group to optical phonon modes. The acoustic-mode energy is primarily determined by the mass of the heavier element. For longitudinal modes at the [001] zone boundaries, where van Hove singularities give rise to a large contribution to $\mathrm{F}(\hbar \omega),{ }^{45}$ the acoustic phonon energy $\hbar \omega_{\mathrm{ac}}=\hbar\left(2 \mathrm{C} / \mathrm{m}_{\mathrm{Me}}\right)^{1 / 2}$, in which $\mathrm{m}_{\mathrm{Me}}$ is the mass of the metal atom and $\mathrm{C}$ is a material-dependent interatomic force constant. ${ }^{46}$ The softening of $\alpha_{\mathrm{tr}}{ }^{2} \mathrm{~F}(\hbar \omega)$ acoustic modes in Figure 3 from $\mathrm{TiN}$ to $\mathrm{ZrN}$ to $\mathrm{CeN}$ to $\mathrm{HfN}$, and the corresponding increase in the energy separation between acoustic and optic mode positions, results primarily from increases in the cation atomic mass: $\mathrm{m}_{\mathrm{Ti}}=47.9, \mathrm{~m}_{\mathrm{Zr}}=91.2$, $\mathrm{m}_{\mathrm{Ce}}=140.1$, and $\mathrm{m}_{\mathrm{Hf}}=178.5 \mathrm{amu}$.

The energy of the optical mode, $\hbar \omega_{\mathrm{op}}=\hbar\left(2 \mathrm{C} / \mathrm{m}_{\mathrm{N}}\right)^{1 / 2}$, is governed by the mass of the lighter element $\left(\mathrm{m}_{\mathrm{N}}=14 \mathrm{amu}\right)$. For the TM nitrides, we obtain interatomic force constants $\mathrm{C}$ which are within $25 \%$ of each other: $\mathrm{C}=87(\mathrm{TiN}), 97(\mathrm{ZrN})$, and $110 \mathrm{~N}-\mathrm{m}^{-1}$ (HfN). For the RE nitride CeN, however, $\mathrm{C}=41 \mathrm{~N}-\mathrm{m}^{-1}$, less than half the average TM nitride $\mathrm{C}$ value, $96 \mathrm{~N}-\mathrm{m}^{-1}$. This is consistent with the fact that the cohesive energy $\mathrm{E}_{\mathrm{coh}}$ of $\mathrm{CeN}, 12.81 \mathrm{eV} /$ formula-unit, ${ }^{47}$ is significantly less than that of the TM nitrides: 14.34 for $\mathrm{TiN}^{48} 15.04$ for $\mathrm{ZrN},{ }^{49}$ and $15.22 \mathrm{eV} /$ formula-unit for HfN. ${ }^{50}$

Measured optical/acoustic $\alpha_{\mathrm{tr}}{ }^{2} \mathrm{~F}(\hbar \omega)$ peak intensity ratios $\mathrm{I}_{\mathrm{op}} / \mathrm{I}_{\mathrm{ac}}$ are $1.2 \pm 0.1 \quad(\mathrm{TiN}), \quad 1.12 \pm 0.05 \quad(\mathrm{ZrN})$, $1.11 \pm 0.05(\mathrm{HfN})$, and $1.18 \pm 0.05(\mathrm{CeN})$. Tunneling spectroscopy measurements ${ }^{12}$ and ab initio $\alpha^{2} \mathrm{~F}(\hbar \omega)$ calculations ${ }^{15}$ for $\mathrm{ZrN}$ are in good agreement with $\mathrm{I}_{\mathrm{op}} / \mathrm{I}_{\mathrm{ac}}=1.25$. For binary compounds with equal acoustic and optical phonon densities and an energy-independent coupling strength $\alpha_{\mathrm{tr}}^{2} \mathrm{~F}(\hbar \omega), \mathrm{I}_{\mathrm{op}} / \mathrm{I}_{\mathrm{ac}}=1$. The proximity of our measured $\mathrm{I}_{\mathrm{op}} / \mathrm{I}_{\mathrm{ac}}$ ratios to unity demonstrates that $\alpha_{\mathrm{tr}}^{2}(\hbar \omega)$ is approximately constant over $\hbar \omega$; thus, the transport Eliashberg spectral function provides, for the compounds investigated here, an effective measurement of the phonon density-of-states.

\section{CONCLUSIONS}

We have determined the transport electron/phonon coupling parameters $\lambda_{\text {tr }}$ and Eliashberg spectral functions $\alpha_{\mathrm{tr}}^{2} \mathrm{~F}(\hbar \omega)$ for stoichiometric epitaxial group-IV TM nitrides $\mathrm{TiN}, \mathrm{ZrN}$, and $\mathrm{HfN}$ and the RE nitride $\mathrm{CeN}$ using an inversion procedure based upon temperature-dependent resistivity measurements $(4 \leq \mathrm{T} \leq 300 \mathrm{~K})$. We find that the coupling parameter is largest for $\mathrm{ZrN}\left(\lambda_{\mathrm{tr}}=1.11\right)$, which exhibits the highest superconducting transition temperature $\mathrm{T}_{\mathrm{c}}=10.4 \mathrm{~K}$, and smallest for $\mathrm{CeN}\left(\lambda_{\mathrm{tr}}=0.44\right)$, which is not superconducting above $4 \mathrm{~K}$. For $\mathrm{HfN}\left(\mathrm{T}_{\mathrm{c}}=9.18 \mathrm{~K}\right)$ and $\mathrm{TiN}\left(\mathrm{T}_{\mathrm{c}}=5.35 \mathrm{~K}\right)$, $\lambda_{\text {tr }}$ is 0.82 and $0.73 . \alpha_{\text {tr }}^{2} \mathrm{~F}(\hbar \omega)$ vs. $\hbar \omega$ results for all compounds investigated indicate that the electron/phonon coupling strength $\alpha_{\text {tr }}^{2}(\hbar \omega)$ is distributed approximately uniformly over energy $\hbar \omega$ and thus the group-IV nitride transport Eliashberg spectral functions provide an effective measure of the phonon density-of-states. The acoustic phonon modes soften monotonically with increasing cation mass from $33 \pm 5$ (TiN) to $25 \pm 2(\mathrm{ZrN})$ to $21 \pm 2(\mathrm{CeN})$ to $18 \mathrm{meV}(\mathrm{HfN})$. The spectral $\alpha_{\mathrm{tr}}{ }^{2} \mathrm{~F}(\hbar \omega)$ peaks corresponding to optical modes in the group-IV TM nitrides remain constant at $\sim 60 \mathrm{meV}(57 \pm 13$ for $\mathrm{TiN}, 60 \pm 6$ for $\mathrm{ZrN}$, and $64 \pm 6 \mathrm{meV}$ for $\mathrm{HfN}$ ) and decrease to $40 \pm 3 \mathrm{meV}$ for the group-IV RE nitride $\mathrm{CeN}$, reflecting a lower bond strength.

\section{ACKNOWLEDGMENTS}

The authors thank Professors Alain Junod, Daniel Gall, and James Eckstein for stimulating discussions. The financial support of the Swedish Research Council (VR) and the Swedish Government Strategic Research Area Grant in Materials Science (SFO Mat-LiU) on Advanced Functional Materials is greatly appreciated. This work was carried out in part in the Frederick Seitz Materials Research Laboratory Central Facilities, University of Illinois.

${ }^{1}$ G. Aschermann, E. Friedrich, E. Justi, and J. Kramer, Phys. Z. 42, 349 (1941).

${ }^{2}$ L. J. Swenson, P. K. Day, B. H. Eom, H. G. Leduc, N. Llombart, C. M. McKenney, O. Noroozian, and J. Zmuidzinas, J. Appl. Phys. 113, 104501 (2013).

${ }^{3}$ H. G. Leduc, B. Bumble, P. K. Day, B. H. Eom, J. Gao, S. Golwala, B. A. Mazin, S. McHugh, A. Merrill, D. C. Moore, O. Noroozian, A. D. Turner, and J. Zmuidzinas, Appl. Phys. Lett. 97, 102509 (2010).

${ }^{4}$ M. R. Vissers, J. Gao, D. S. Wisbey, D. A. Hite, C. C. Tsuei, A. D. Corcoles, M. Steffen, and D. P. Pappas, Appl. Phys. Lett. 97, 232509 (2010).

${ }^{5}$ R. D. Leo, A. Nigro, G. Nobile, and R. Vaglio, J. Low Temp. Phys. 78, 41 (1990).

${ }^{6}$ R. Kampwirth, D. W. Capone II, K. E. Gray, and A. Vicens, IEEE Trans. Magn. 21, 459 (1985).

${ }^{7}$ J. B. Chang, M. R. Vissers, A. D. Córcoles, M. Sandberg, J. Gao, D. W. Abraham, J. M. Chow, J. M. Gambetta, M. Beth Rothwell, G. A. Keefe, M. Steffen, and D. P. Pappas, Appl. Phys. Lett. 103, 012602 (2013).

${ }^{8}$ K. Schwarz, A. R. Williams, J. J. Cuomo, J. H. E. Harper, and H. T. Hentzell, Phys. Rev. B 32, 8312 (1985).

${ }^{9}$ P. Khosropanah, J. R. Gao, W. M. Laauwen, M. Hajenius, and T. M. Klapwijk, Appl. Phys. Lett. 91, 221111 (2007).

${ }^{10}$ S. Miki, M. Fujiwara, M. Sasaki, B. Baek, A. J. Miller, R. H. Hadfield, S. W. Nam, and Z. Wang, Appl. Phys. Lett. 92, 061116 (2008).

${ }^{11}$ N. N. Iosad, V. V. Roddatis, S. N. Polyakov, A. V. Varlashkin, B. D. Jackson, P. N. Dmitriev, J. R. Gao, and T. M. Klapwijk, IEEE Trans. Appl. Supercond. 11, 3832 (2001).

${ }^{12}$ J. Geerk, G. Linker, and R. Smithey, Phys. Rev. Lett. 57, 3284 (1986)

${ }^{13}$ S. Yamanaka, K. Hotehama, and H. Kawaji, Nature 392, 580 (1998).

${ }^{14}$ B. Sacépé, C. Chapelier, T. I. Baturina, V. M. Vinokur, M. R. Baklanov, and M. Sanquer, Nature Commun. 1, 140 (2010).

${ }^{15}$ R. Heid, K.-P. Bohnen, B. Renker, T. Wolf, and H. Schober, Phys. Rev. B 71, 092302 (2005).

${ }^{16}$ W. L. McMillan, Phys. Rev. 167, 331 (1968).

${ }^{17}$ D. J. Scalapino and P. W. Anderson, Phys. Rev. 133, A921 (1964).

${ }^{18}$ G. Grimvall, The Electron-phonon Interaction in Metals (North-Holland Publishing, Co., sole distributors for the U.S.A. and Canada, Elsevier, North-Holland, 1981).

${ }^{19}$ I. Petrov, F. Adibi, J. E. Greene, W. D. Sproul, and W.-D. Munz, J. Vac. Sci. Technol. A 10, 3283 (1992).

${ }^{20}$ A. B. Mei, B. M. Howe, C. Zhang, M. Sardela, J. N. Eckstein, L. Hultman, A. Rockett, I. Petrov, and J. E. Greene, J. Vac. Sci. Technol. 31, 061516 (2013).

${ }^{21}$ H. S. Seo, T. Y. Lee, J. G. Wen, I. Petrov, J. E. Greene, and D. Gall, J. Appl. Phys. 96, 878 (2004).

${ }^{22}$ T.-Y. Lee, D. Gall, C.-S. Shin, N. Hellgren, I. Petrov, and J. E. Greene, J. Appl. Phys. 94, 921 (2003). 
${ }^{23}$ A. Guinier, X-ray Diffraction in Crystals, Imperfect Crystals, and Amorphous Bodies (Dover, New York, 1994), p. 124.

${ }^{24}$ L. J. van der Pauw, Philips Res. Rep. 13, 1 (1958).

${ }^{25}$ CRC Materials Science and Engineering Handbook, edited by J. F. Shackelford and W. Alexander, 3rd ed. (CRC Press, 2000).

${ }^{26}$ H. O. Pierson, Handbook of Refractory Carbides \& Nitrides: Properties, Characteristics, Processing and Applications 1st ed. (William Andrew, 1997).

${ }^{27}$ N. Kumar, K. Pourrezaei, M. Fissel, T. Begley, B. Lee, and E. C. Douglas, J. Vac. Sci. Technol. A 5, 1778 (1987).

${ }^{28}$ H. Norström, S. Nygren, P. Wiklund, M. Östling, R. Buchta, and C. S. Petersson, Vacuum 35, 547 (1985).

${ }^{29}$ A. Rizzo, M. A. Signore, D. Valerini, D. Altamura, A. Cappello, and L. Tapfer, Surf. Coat. Technol. 206, 2711 (2012).

${ }^{30}$ S. Norouzian, M. M. Larijani, and R. Afzalzadeh, Bull. Mater. Sci. 35, 885 (2012).

${ }^{31}$ S. A. Barnett, L. Hultman, J.-E. Sundgren, F. Ronin, and S. Rohde, Appl. Phys. Lett. 53, 400 (1988).

${ }^{32}$ B. O. Johansson, J.-E. Sundgren, and U. Helmersson, J. Appl. Phys. 58, 3112 (1985).

${ }^{33}$ S. Q. Xiao, K. Tsuzuki, H. Sugimura, and O. Takai, J. Mater. Sci. 33, 3251 (1998).

${ }^{34}$ S. Q. Xiao and O. Takai, Thin Solid Films 317, 137 (1998).

${ }^{35}$ S. Q. Xiao, K. Tsuzuki, C. P. Lungu, and O. Takai, Vacuum 51, 691 (1998).

${ }^{36} \mathrm{M}$. Lundstrom, Fundamentals of Carrier Transport, 2nd ed. (Cambridge University Press, 2009).

${ }^{37}$ G. v. Minnigerode, Ber. Bunsenges. Phys. Chem. 87, 453 (1983).

${ }^{38}$ G. Eliashberg, Sov. Phys. JETP 11, 696 (1960).

${ }^{39} \mathrm{G}$. Grimvall, The Electron-phonon Interaction in Metals (North-Holland Pub. Co.: Sole distributors for the U.S.A. and Canada, Elsevier NorthHolland, Amsterdam; New York, 1981).
${ }^{40}$ P. Giannozzi, S. Baroni, N. Bonini, M. Calandra, R. Car, C. Cavazzoni, D. Ceresoli, G. L. Chiarotti, M. Cococcioni, I. Dabo, A. Dal Corso, S. de Gironcoli, S. Fabris, G. Fratesi, R. Gebauer, U. Gerstmann, C. Gougoussis, A. Kokalj, M. Lazzeri, L. Martin-Samos, N. Marzari, F. Mauri, R. Mazzarello, S. Paolini, A. Pasquarello, L. Paulatto, C. Sbraccia, S. Scandolo, G. Sclauzero, A. P. Seitsonen, A. Smogunov, P. Umari, and R. M. Wentzcovitch, J. Phys. Condens. Matter 21, 395502 (2009).

${ }^{41}$ G. K. H. Madsen and D. J. Singh, Comput. Phys. Commun. 175, 67 (2006).

${ }^{42}$ R. Lortz, Y. Wang, S. Abe, C. Meingast, Y. B. Paderno, V. Filippov, and A. Junod, Phys. Rev. B 72, 024547 (2005).

${ }^{43}$ V. Kanchana, G. Vaitheeswaran, X. Zhang, Y. Ma, A. Svane, and O. Eriksson, Phys. Rev. B 84, 205135 (2011)

${ }^{44}$ M. Stoehr, C.-S. Shin, I. Petrov, and J. E. Greene, J. Appl. Phys. 110, 083503 (2011).

${ }^{45}$ N. W. Ashcroft and N. D. Mermin, Solid State Physics, 1st ed. (BrooksCole, 1976).

${ }^{46}$ C. Kittel, Introduction to Solid State Physics, 7 th ed. (Wiley, 1995).

${ }^{47}$ E. Voloshina and B. Paulus, J. Comput. Chem. 29, 2107 (2008).

${ }^{48}$ D. Gall, S. Kodambaka, M. A. Wall, I. Petrov, and J. E. Greene, J. Appl. Phys. 93, 9086 (2003).

${ }^{49}$ A. F. Guillermet, J. Häglund, and G. Grimvall, Phys. Rev. B 45, 11557 (1992).

${ }^{50}$ A. F. Guillermet, J. Häglund, and G. Grimvall, Phys. Rev. B 48, 11673 (1993).

${ }^{51} \lambda_{\text {tr }}$ differs from the McMillian electron/phonon coupling parameter $\lambda$ by the weighted geometric factor $(1-\cos \theta)$, in which $\cos \theta=\mathbf{k} \cdot \mathbf{k}^{\prime} /|\mathbf{k}|\left|\mathbf{k}^{\prime}\right|$ and $\theta$ is the angle formed by the electron wave vector prior $\mathbf{k}$ and subsequent $\mathbf{k}^{\prime}$ to scattering. ${ }^{15,40} \mathrm{C}$. Poole et al. ${ }^{40}$ conclude, "the difference is typically no more than $15 \%$, and never as much as a factor of $2 . "$ 